\title{
How Well Does the Surprise Question Predict 1-year Mortality for Patients Admitted with COPD?
}

\author{
Dana Tripp, $B A^{7}$, Jaclyn Janis, $M P H, B S N, R N^{2}$, Benjamin Jarrett, $M D, M P H^{3}$, \\ F. Lee Lucas, $P h D^{2}$, Tania D. Strout, PhD, RN, MS ${ }^{7,4}$, Paul K. J. Han, MD MA MPH ${ }^{1,2}$, \\ Isabella Stumpf, $D O^{1,5}$, and Rebecca N. Hutchinson, $M D, M P H^{1,2,5}$ (D)
}

\begin{abstract}
${ }^{1}$ Tufts University School of Medicine, Boston, MA, USA; ${ }^{2}$ Center for Outcomes Research and Evaluation, Maine Medical Center Research Institute, Portland, ME, USA; ${ }^{3}$ Division of Pulmonary Medicine, University of Arizona Health Sciences, Tucson, AZ, USA; ${ }^{4}$ Department of Emergency Medicine, Maine Medical Center, Portland, ME, USA; ${ }^{5}$ Division of Palliative Medicine, Maine Medical Center, Portland, ME, USA.
\end{abstract}

BACKGROUND: Patients with chronic obstructive pulmonary disease (COPD) often receive burdensome care at end-of-life (EOL) and infrequently complete advance care planning (ACP). The surprise question (SQ) is a prognostic tool that may facilitate ACP.

OBJECTIVE: To assess how well the SQ predicts mortality and prompts ACP for COPD patients.

DESIGN: Retrospective cohort study.

SUBJECTS: Patients admitted to the hospital for an acute exacerbation of COPD between July 2015 and September 2018.

MAIN MEASURES: Emergency department (ED) and inpatient clinicians answered, "Would you be surprised if this patient died in the next 30 days (ED)/one year (inpatient)?" The primary outcome measure was the accuracy of the SQ in predicting 30-day and 1-year mortality. The secondary outcome was the correlation between SQ and ACP (palliative care consultation, documented goals-ofcare conversation, change in code status, or completion of ACP document).

KEY RESULTS: The 30-day SQ had a high specificity but low sensitivity for predicting 30-day mortality: sensitivity 12\%, specificity 95\%, PPV 11\%, and NPV 96\%. The 1-year SQ demonstrated better accuracy for predicting 1-year mortality: sensitivity $47 \%$, specificity $75 \%$, PPV $35 \%$, and NPV 83\%. After multivariable adjustment for age, sex, and prior 6-month admissions, 1-year SQ+ responses were associated with greater odds of 1-year mortality (OR 2.38, 95\% CI 1.39-4.08) versus SQ-. One-year $\mathrm{SQ}+$ patients were more likely to have a goals-of-care conversation (25\% vs. $11 \%, p<0.01)$ and complete an advance directive or POLST (46\% vs. $23 \%, p<0.01$ ). After multivariable adjustment, $\mathrm{SQ}+$ responses to the 1-year SQ were associated with greater odds of ACP receipt (OR 2.67, 95\% CI 1.64-4.36).

CONCLUSIONS: The 1-year surprise question may be an effective component of prognostication and advance care planning for COPD patients in the inpatient setting.

Received July 29, 2020

Accepted December 17, 2020

Published online January 6, 2021
KEY WORDS: prognostication; goals-of-care; advance care planning; surprise question; chronic obstructive pulmonary disease.

J Gen Intern Med 36(9):2656-62

DOI: $10.1007 / \mathrm{s} 11606-020-06512-8$

(C) Society of General Internal Medicine 2021

\section{INTRODUCTION}

Chronic obstructive pulmonary disease (COPD), the fourth leading cause of death globally, ${ }^{1,2}$ is a progressive disease with debilitating symptoms. ${ }^{3-5}$ COPD patients have end-oflife (EOL) treatment preferences similar to those of lung cancer patients, yet higher rates of burdensome care such as mechanical ventilation and cardiopulmonary resuscitation. ${ }^{6-8}$ Advance care planning (ACP) and specialty palliative care decrease nonbeneficial aggressive care; however, COPD patients have low rates of $\mathrm{ACP}{ }^{9-11}$ and palliative care involvement. ${ }^{7,8,12,13}$ The poorer quality of EOL care for COPD patients may be due in part to the increased prognostic uncertainty associated with the disease course. ${ }^{14}$ Therefore, an accurate and easy-to-use prognostic tool may facilitate efforts to improve EOL care for COPD patients.

Current validated models for predicting death in COPD are dependent on pulmonary function testing (PFT) or the 6-min walk test, ${ }^{15,16}$ often inaccessible for the inpatient provider. Prognostic indices without PFT data are limited by their use of dyspnea, a symptom that is difficult to measure reliably. ${ }^{17}$ Risk calculators have been developed to predict in-hospital mortality, ${ }^{18}$ but are typically applied too late in the disease process to promote helpful ACP. Laboratory tests such as thrombocytosis or C-reactive protein do not reliably predict 1-year mortality. ${ }^{16,19}$

Studies in other diseases such as cancer, kidney disease, and heart failure have found that the surprise question (SQ) can predict mortality. ${ }^{20-32}$ The SQ prompts clinicians to answer, "Would you be surprised if this patient died in the next [time period]?" When applied to a heterogeneous population in the primary care or ED setting, the SQ is not as predictive. ${ }^{22,24}$ There is limited data about the utility of the SQ for COPD patients. Prior studies have looked at the SQ's predictive 
accuracy in COPD relative to other prognostic indicators, such as the Gold Standards Framework Indicator Guidance, rather than mortality. ${ }^{33,34}$

We conducted a quality improvement initiative with the overarching goal to use the SQ to enhance the delivery of care for inpatients with COPD by engaging them in ACP. As part of this initiative, we sought to evaluate how well the 30-day and 1-year SQs predict mortality in patients admitted for an acute exacerbation of COPD. We also investigated whether the SQ was associated with increases in ACP.

\section{METHODS}

\section{Study Design, Setting, and Population}

This study was part of a quality improvement initiative to increase ACP and palliative medicine involvement for COPD patients. We performed a retrospective study of all patients admitted to our 637-bed tertiary care hospital for an acute exacerbation of COPD between July 2015 and September 2018. Patients were identified using the methodology described by the Yale Center for Outcomes Research and Evaluation. ${ }^{35}$ We restricted our sample to patients with primary residence in Maine or New Hampshire in order to increase the likelihood of obtaining complete follow-up data. Trained abstractors were blind to SQ response and used a data extraction tool designed in REDCap (Research Electronic Data Capture). ${ }^{36}$ The study was reviewed by the Maine Medical Center IRB and approved as quality improvement. Data is available upon request.

\section{Patient and Admission Characteristics}

We abstracted the following demographic data: age, sex, race/ethnicity, zip code of residence, insurer, comorbidities, and number of all-cause admissions 6 months prior to index admission. Primary insurance was categorized by payer as Medicare, Medicaid, commercial, Veterans Affairs, or other (including self-pay and worker's compensation). We obtained characteristics of the index admission including disposition and number of readmissions within the subsequent six months.

\section{Surprise Question}

Attending physicians, resident physicians, and advanced practice providers answered one of two SQs, depending on the site of care. ED clinicians answered, "Would you be surprised if this patient died in the next 30 days?" whereas inpatient clinicians answered, "Would you be surprised if this patient died in the next year?" Clinicians provided a binary response of "No, I would not be surprised" (positive, SQ+) or "Yes, I would be surprised" (negative, SQ-). The SQ was mandatory for ED providers at the time of admission and for inpatient providers if they used the general inpatient medicine admission order set. A positive 30-day SQ results in an automatically checked palliative care consultation; a positive 1-year SQ triggers a suggestion for palliative care. None of the researchers were clinicians who answered the SQ. Responses to the SQ were not communicated between clinicians across settings.

\section{Outcomes}

Our primary outcomes were 30-day and 1-year mortality. In order to maximize the completeness of mortality data, we used multiple sources. First, we collected mortality data from our EMR, which includes outpatient facilities and hospitals throughout Maine. If there was no evidence that the patient was deceased, we looked for notes in our EMR indicating that the patient lived beyond our follow-up period of 1 year. We then searched in HealthInfoNet, a health information exchange that links medical information from the majority of health care sites in Maine. ${ }^{37}$ Finally, we performed online obituary searches.

Our secondary outcome was ACP completion. The composite ACP outcome included any of newly scanned or completed AD (advance directive) or POLST (Physician Orders for Life Sustaining Therapy) within 6 months of admission, a goals-of-care conversation, palliative care consultation, or change in code status during the hospital stay. We chose this definition of ACP based on expert consensus definition. ${ }^{38} \mathrm{We}$ defined a goals-of-care conversation as documentation by a physician or APP of engaging the patient or surrogate decision-maker in discussing preferences for treatment including code status, wishes for life-sustaining therapies, or appropriateness of specific medical therapies. ${ }^{39}$ All charts were reviewed manually using a search function in EPIC with unambiguous terms and by reviewing provider notes. Ninety-one charts $(18.2 \%)$ were abstracted by two members of the team with agreement on all but three (3\%, kappa statistic 0.93 ). A senior member of the team served as the adjudicator for those three charts. AD/POLST data was obtained from the $\mathrm{ACP}$ tab in EPIC.

\section{Statistical Analysis}

We assessed demographic and admission characteristics by responses to the SQ. Data were dichotomized into two groups using responses to the SQ ("No, I would not be surprised" [SQ+] or "Yes, I would be surprised" [SQ-]). We then compared characteristics for the groups using the chi-square statistic with Yates' continuity correction or two-tailed Fisher's exact test. We assessed the accuracy of the SQ in predicting 30-day mortality, 1-year mortality, and ACP by calculating sensitivity, specificity, positive predictive value (PPV), negative predictive value (NPV), positive likelihood ratio $(+\mathrm{LR})$, and negative likelihood ratio (-LR) using the epiR package in $\mathrm{R}^{40}$

We used unadjusted logistic regression to evaluate the association between the 30-day SQ and 30-day mortality and $\mathrm{ACP}$; we did not adjust for covariates due to the rarity of the 
outcome and the potential for overfitting. We used unadjusted and adjusted logistic regression to evaluate the association between the 1-year SQ and 1-year mortality. We decided, $a$ priori, to control for age and sex in mortality outcome models. We aimed for a parsimonious model by selecting covariates based on significant associations with mortality or changes in odds ratios of greater than $10 \%$. Therefore, the final covariateadjusted model for 1-year mortality included age, sex, and number of admissions in the prior 6 months. In addition, we used logistic regression to evaluate the association between responses to the SQs and the presence of ACP. We decided, $a$ priori, to control for age, sex, and number of admissions in the previous 6 months for the 1-year SQ because of clinical significance, as multiple admissions provide more opportunity for ACP. Statistical significance was set at $p<0.05$. All analyses were performed in $\mathrm{R}$ (version 3.5 .1 ). ${ }^{41}$

\section{RESULTS}

\section{Population Characteristics}

A total of 439 patients were admitted for an acute exacerbation of COPD between July 2015 and September 2018 and had a documented response to either the 30-day or 1-year SQ. Patients were excluded for incomplete medical records (patient lived in a different state at the time of data collection or no medical records in EMR since discharge, $n=8$ ), and incomplete death data (EMR stated deceased but date/location of death unavailable in EMR/other sources, or last EMR note was a transfer to hospice with no other data, $n=3$ ). Our final cohort included 428 patients. A total of 381 patients had responses to the 30-day SQ; 365 patients had responses to the one-year SQ. There were no differences in baseline characteristics between our overall cohort, those who had responses to the 30-day SQ, and those who had responses to the 1-year SQ (data not shown). Five percent $(n=19)$ of 30 day SQ responses were $\mathrm{SQ}+$, and $30 \%(n=108)$ of 1 -year responses were $\mathrm{SQ}+$.

Thirty-day SQ+ patients were older than 30-day SQ- patients and 1-year SQ+ patients were older than 1-year SQpatients (Table 1). The overall comorbidity burden was similar for both the 30-day and 1-year SQ+ patients as compared to SQ-. Greater proportions of 1-year SQ+ patients were admitted one or more times in the prior 6 months as compared to SQ- patients ( $45 \%$ vs. $24 \%, p<0.001)$ (Table 1$)$. The number of admissions in the prior 6 months did not differ based on the

Table 1 Patient Characteristics Overall and by Response to 30-Day and 1-Year Surprise Questions

\begin{tabular}{|c|c|c|c|c|c|}
\hline & \multirow[b]{3}{*}{ Total } & \multicolumn{2}{|c|}{ 30-day surprise question } & \multicolumn{2}{|c|}{ 1-year surprise question } \\
\hline & & \multicolumn{2}{|l|}{$(n=381)$} & \multicolumn{2}{|l|}{$(n=365)$} \\
\hline & & \multirow{2}{*}{$\frac{S Q+{ }^{a}}{(n=19)}$} & \multirow{2}{*}{$\frac{\text { SQ- }^{b}}{(n=362)}$} & \multirow{2}{*}{$\frac{\mathrm{SQ}^{+{ }^{\mathrm{a}}}}{(n=108)}$} & \multirow{2}{*}{$\frac{\mathrm{SQ}^{\mathrm{b}}}{(n=257)}$} \\
\hline & $(n=428)$ & & & & \\
\hline \multicolumn{6}{|l|}{ Age category } \\
\hline$<=55$ & $52(12 \%)$ & $1(5 \%)$ & $47(13 \%)$ & $9(8 \%)$ & $35(14 \%)$ \\
\hline $56-65$ & $115(27 \%)$ & $1(5 \%)$ & $98(27 \%)$ & $30(28 \%)$ & $66(26 \%)$ \\
\hline $66-75$ & $117(27 \%)$ & $7(37 \%)$ & $98(27 \%)$ & $28(26 \%)$ & $70(27 \%)$ \\
\hline $76-85$ & $105(25 \%)$ & $6(32 \%)$ & $89(25 \%)$ & $26(24 \%)$ & $66(26 \%)$ \\
\hline $85+$ & $39(9 \%)$ & $4(21 \%)$ & $30(8 \%)$ & $15(14 \%)$ & $20(8 \%)$ \\
\hline Female & $218(51 \%)$ & $11(58 \%)$ & $182(50 \%)$ & $63(58 \%)$ & $123(48 \%)$ \\
\hline Non-Hispanic white & $419(98 \%)$ & $19(100 \%)$ & $353(98 \%)$ & $106(98 \%)$ & $251(98 \%)$ \\
\hline \multicolumn{6}{|l|}{ Insurer } \\
\hline Medicare & $316(74 \%)$ & $17(89 \%)$ & $264(73 \%)$ & $82(76 \%)$ & $192(75 \%)$ \\
\hline Medicaid & $39(9 \%)$ & $1(5 \%)$ & $32(9 \%)$ & $13(12 \%)$ & $21(8 \%)$ \\
\hline Commercial & $49(11 \%)$ & $1(5 \%)$ & $44(12 \%)$ & $6(6 \%)$ & $29(11 \%)$ \\
\hline VA & $10(2 \%)$ & $0(0 \%)$ & $9(2 \%)$ & $4(4 \%)$ & $6(2 \%)$ \\
\hline Self/other & $14(3 \%)$ & $0(0 \%)$ & $13(4 \%)$ & $3(3 \%)$ & $9(4 \%)$ \\
\hline \multicolumn{6}{|l|}{ Comorbidities } \\
\hline $\mathrm{CHF}$ & $149(35 \%)$ & $11(58 \%)^{\mathrm{d}}$ & $117(32 \%)^{\mathrm{d}}$ & $36(33 \%)$ & $86(33 \%)$ \\
\hline Diabetes & $118(28 \%)$ & $8(42 \%)$ & $99(27 \%)$ & $24(22 \%)$ & $73(28 \%)$ \\
\hline Pulmonary diseasec & $38(9 \%)$ & $4(21 \%)$ & $33(9 \%)$ & $11(10 \%)$ & $18(7 \%)$ \\
\hline Dementia & $30(7 \%)$ & $3(16 \%)$ & $19(5 \%)$ & $11(10 \%)$ & $14(5 \%)$ \\
\hline CVA & $53(12 \%)$ & $3(16 \%)$ & $44(12 \%)$ & $14(13 \%)$ & $29(11 \%)$ \\
\hline CKD & $106(25 \%)$ & $6(32 \%)$ & $82(23 \%)$ & $33(31 \%)$ & $54(21 \%)$ \\
\hline HTN & $304(71 \%)$ & $17(89 \%)$ & $250(69 \%)$ & $84(78 \%)$ & $177(69 \%)$ \\
\hline CAD & $145(34 \%)$ & $9(47 \%)$ & $119(33 \%)$ & $49(45 \%)^{\mathrm{d}}$ & $70(27 \%)^{\mathrm{d}}$ \\
\hline Lung/airway cancer & $40(9 \%)$ & $4(21 \%)$ & $31(9 \%)$ & $11(10 \%)$ & $21(8 \%)$ \\
\hline Non-respiratory malignancy & $57(13 \%)$ & $1(5 \%)$ & $51(14 \%)$ & $20(19 \%)$ & $32(12 \%)$ \\
\hline \multicolumn{6}{|c|}{ Number of admissions in prior 6 months } \\
\hline None & $301(70 \%)$ & $10(53 \%)$ & $257(71 \%)$ & $59(55 \%)^{\mathrm{d}}$ & $196(76 \%)^{\mathrm{d}}$ \\
\hline $1+$ & $127(30 \%)$ & $9(47 \%)$ & $105(29 \%)$ & $49(45 \%)$ & $61(24 \%)$ \\
\hline
\end{tabular}

${ }^{a} S Q+$ response equates, "No, would not be surprised"

"SQ- response equates, "Yes, would be surprised"

"Interstitial lung disease and/or pulmonary hypertension

${ }^{d}$ Statistically significant for $S Q+$ versus $S Q-$ within 30-day or 1-year surprise question at $p<0.05$ 
30-day SQ. More 1-year SQ- patients were discharged home compared to SQ+ (81\% vs. $66 \%)$, and more 1-year SQ+ patients were discharged to a skilled nursing facility or inpatient rehabilitation compared to SQ- (29\% vs. 16\%) (Supplemental Table 1). A greater proportion of one-year SQ+ patients were readmitted one or more times within 6 months as compared to SQ- patients (49\% vs. $37 \%, p=$ 0.042 ), but the number of readmissions did not significantly differ based on the 30-day SQ. The proportion of patients who were intubated was similar for both the 30-day and 1-year SQ+ and SQ- patients.

\section{Accuracy of the $S Q$ in Predicting Mortality}

ED providers reported that they would not be surprised if the patient died in the next 30 days in 5\% of cases $(n=19)$. Of these patients, $11 \%(n=2)$ died within 30 days. Inpatient providers reported that they would not be surprised if the patient died in the next year in $30 \%$ of cases $(n=108)$. Of these patients, $35 \%(n=38)$ died within 1 year.

A "No" response to the 30-day SQ (SQ+) had the following accuracy for predicting 30-day mortality (Table 2): sensitivity $12 \%$, specificity $95 \%$, PPV $11 \%$, and NPV $96 \%$. The positive likelihood ratio (LR) was 2.68, and the negative LR was 0.92 . The 1-year SQ had slightly better accuracy for predicting 1year mortality: sensitivity $47 \%$, specificity $75 \%$, PPV $35 \%$, and NPV $83 \%$. The positive LR was 1.90 and the negative LR was 0.70 .

Using unadjusted logistic regression models, we did not find a significant association between the 30-day SQ and 30day mortality (Fig. 1). Patients with a 1-year SQ+ had 2.7 times greater odds of dying within 1 year than those with a SQ(95\% CI: $1.62-4.51 ; p<0.01$ ). After adjustment for age, sex, and prior 6-month admissions, 1-year SQ+ remained associated with greater odds of 1-year mortality (OR 2.38, 95\% CI 1.39-4.08; $p<0.01$; Fig. 1).

\section{Receipt of Advance Care Planning and the SQ}

The occurrence of ACP during index admission among the entire cohort was as follows: goals-of-care conversation, $16 \%$; updated $\mathrm{AD} / \mathrm{POLST}, 31 \%$; any change in code status, $22 \%$; and palliative care consult, $14 \%$ (Table 3 ). More patients with a 30 -day SQ+ had a goals-of-care conversation ( $42 \%$ vs. $15 \%$, $p<0.01$ ), and more received a palliative care consult (47\% vs. $12 \%, p<0.01)$ than patients with a SQ- response. More patients with a 1-year SQ+ had a goals-of-care conversation $(25 \%$ vs. $11 \%, p<0.01)$, a code status change from full code to decreased measures ( $31 \%$ vs. $15 \%, p<0.01)$, and an updated AD/POLST ( $46 \%$ vs. $23 \%, p<0.01$ ) than patients with a 1-year SQ-. In unadjusted models, patients with a 30day SQ+ had 3.87 times greater odds of having received ACP than SQ- patients (95\% CI: $1.26-11.91 ; p=0.02)$. One-year $\mathrm{SQ}+$ patients had 3.09 times greater odds of having received ACP than SQ- patients in unadjusted models (95\% CI: 1.934.94; $p<0.001$ ), and 2.67 times greater odds after adjusting for age, sex, and prior 6-month admissions (95\% CI: 1.64 $4.36, p<0.01$ ) (Fig. 1).

\section{DISCUSSION}

Our findings serve as an important step toward understanding the surprise question's prognostic accuracy for the COPD population and its association with ACP. To our knowledge, this is the first study to assess the prognostic accuracy of both the 30-day and 1-year SQ in patients with COPD. We found that both the 30-day and 1-year SQ had mixed accuracy, with low-to-moderate sensitivity and high specificity. Patients whose providers answered that they would not be surprised if the patient died in the next year were more than two times as likely to die within one year after adjustment for age, sex, and number of prior 6-month admissions. We did not find a significant association between the 30-day SQ and 30-day mortality. The odds of receiving ACP were at least three times greater among those with either a 1-year or 30-day SQ+ compared to their SQ- counterparts; however, the overall occurrence of ACP remained low regardless of the response to the SQ.

Only two other studies have examined the use of the SQ in COPD prognostication. ${ }^{33,34}$ However, these studies used the Gold Standards Framework Prognostic Indicators Guidance (GSF PIG) as the gold standard, rather than mortality, so we

Table 2 Test Characteristics of 30-Day and 1-Year Surprise Questions for Predicting Mortality

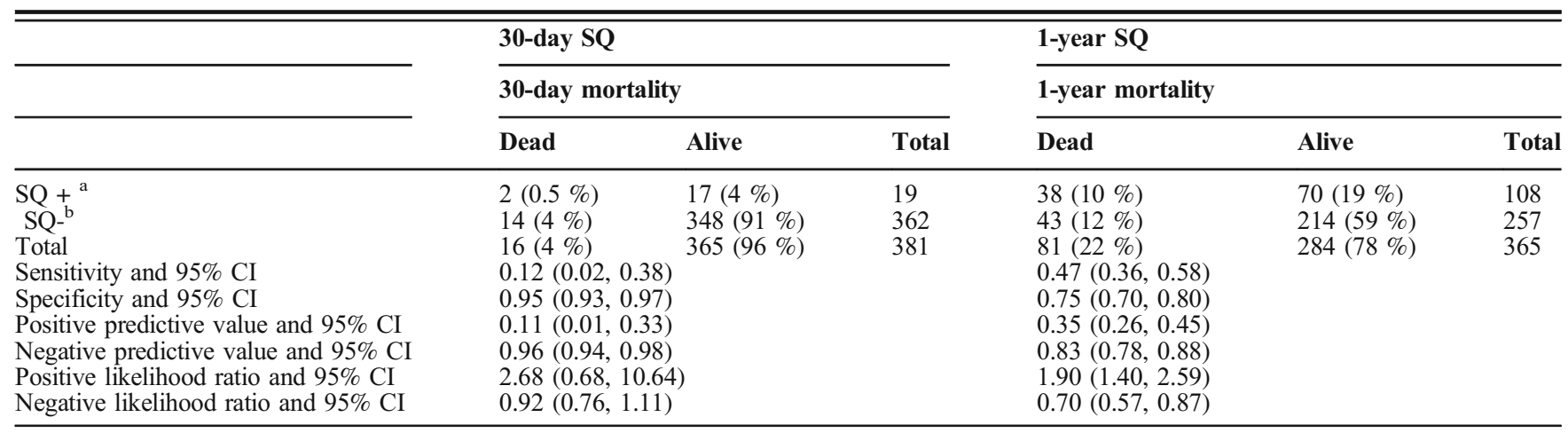




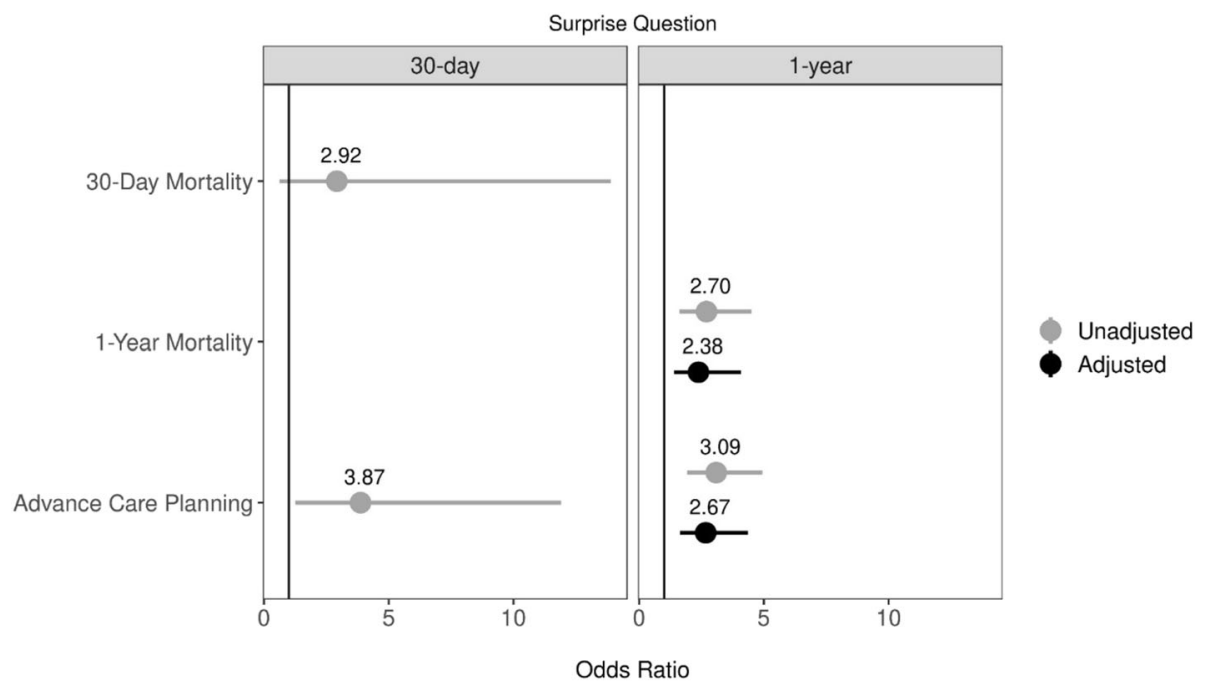

Figure 1 Unadjusted and covariate-adjusted associations of 30-day and 1-year surprise questions and outcomes of mortality and advance care planning. Note: 1-year mortality was adjusted for age, sex, and prior 6-month admissions; 1-year ACP were adjusted for age, sex, and prior 6month admissions.

are unable to compare our findings. We are unaware of other published efforts to assess the accuracy of other commonlyused prognostic tools for COPD, e.g., GOLD and BODE, in predicting 1-year mortality.

Our study suggests that the SQ performs less well for patients with COPD than for patients with cancer. This is similar to prior studies of SQ in chronic diseases, such as renal disease, heart failure, and other respiratory diseases. ${ }^{25,30,42}$ This poorer performance may be due to the more unpredictable course of illness in COPD compared to other diseases. ${ }^{14,42}$ Compared with a recent study of all patients presenting to the ED, our 30-day ED SQ had lower sensitivity ( $32 \%$ vs. $12 \%$ ), higher specificity ( $85 \%$ vs. $95 \%$ ), and similar PPV and NPV. ${ }^{24}$ Our findings also suggest that ED providers are less able to identify COPD patients very near the end-oflife than they are for the general ED population. The reasons behind this difference are not clear but may also be related to
COPD's unpredictable disease course. Despite the test characteristics, our logistic regression models suggest the SQ has additive prognostic value.

This is the first study to examine the association between a positive SQ response and the occurrence of ACP. Our results suggest that $\mathrm{SQ}+$ is associated with an increase in ACP; however, overall occurrence of ACP remains low, leaving substantial opportunity for improvement. Of note, our institution had initiated efforts to increase palliative care consultations, including automatic consult triggers associated with the 30-day SQ+; however, consult orders can be removed and our results indicate palliative care consultation rates remained low. Our findings are consistent with other studies showing that the SQ is not effective in increasing referrals to palliative care for cancer patients, ${ }^{43}$ or for a heterogeneous population of ED patients. ${ }^{24}$ The overall low occurrence of palliative care consultations, goals-of-care conversations, and $\mathrm{AD}$ completion

Table 3 Receipt of Advance Care Planning ${ }^{a}$ by Response to 30-Day and 1-Year Surprise Questions

\begin{tabular}{|c|c|c|c|c|c|c|c|}
\hline & \multirow[b]{2}{*}{ Total } & \multicolumn{2}{|c|}{$\begin{array}{l}\text { 30-day surprise question } \\
(n=381)\end{array}$} & \multirow[t]{3}{*}{$p$ value } & \multicolumn{2}{|c|}{$\begin{array}{l}\text { 1-year surprise question } \\
(n=365)\end{array}$} & \multirow[t]{3}{*}{$p$ value } \\
\hline & & $\mathbf{S Q}+^{\mathbf{b}}$ & $\mathrm{SQ}^{\mathrm{c}}$ & & $\mathbf{S Q}+^{\mathbf{b}}$ & SQ- ${ }^{c}$ & \\
\hline & $(n=428)$ & $(n=19)$ & $(n=362)$ & & $(n=108)$ & $(n=257)$ & \\
\hline Goals of care discussion & $68(16 \%)$ & $8(42 \%)$ & $54(15 \%)$ & $<0.01$ & $27(25 \%)$ & $27(11 \%)$ & $<0.01$ \\
\hline AD/POLST completed & $132(31 \%)$ & $10(53 \%)$ & $115(32 \%)$ & 0.10 & $50(46 \%)$ & $58(23 \%)$ & $<0.01$ \\
\hline Code status change & & & & 0.40 & & & $<0.01$ \\
\hline No change & $335(78 \%)$ & $14(74 \%)$ & $281(78 \%)$ & & $74(69 \%)$ & $214(83 \%)$ & \\
\hline Full to decreased measures & $85(20 \%)$ & $4(21 \%)$ & $74(20 \%)$ & & $33(31 \%)$ & $38(15 \%)$ & \\
\hline Decreased measures to full/increased & $5(1 \%)$ & $1(5.3 \%)$ & $4(1.1 \%)$ & & $1(1 \%)$ & $2(1 \%)$ & \\
\hline Palliative care consult & $83(19 \%)$ & $9(47 \%)$ & $45(12 \%)$ & $<0.01$ & $19(18 \%)$ & $27(11 \%)$ & 0.09 \\
\hline
\end{tabular}

${ }^{a}$ Advance care planning was considered to have occurred if any of the following were true: advance directive or physician orders for life sustaining treatment form in a chart during or after admission, goals of care discussion completed, palliative care consultation performed, or any change in code status documented

${ }^{b} S Q+$ response equates, "No, would not be surprised"

"SQ- response equates, "Yes, would be surprised" 
are consistent with prior studies of $\mathrm{COPD}^{10,11}$ and other diseases. $^{7,8,13,33}$ These findings, however, are inconsistent with a survey in which $55 \%$ of physicians stated that SQ+ response prompts $\mathrm{ACP}{ }^{44}$ It is not clear why the SQ was ineffective in triggering $\mathrm{ACP}$ or palliative care consultation. The utility of the SQ in prompting ACP is predicated on the premise that it initiates a process of pause, reflection, and action by the provider. ${ }^{45,46}$ Providers must also believe in the accuracy of the SQ in order for it to affect their care.

Our study has several limitations. Our cohort includes mostly non-Hispanic White patients from a single institution in Maine, which may limit its generalizability to more diverse populations. Although we made substantive efforts to ensure complete death data, it is possible that some patients who died were missed. In our institution, the SQ is answered by providers with varying training and experience, which may be associated with the accuracy of the SQ. In order to assess presence of goals-of-care conversations, we performed manual chart abstractions. We used multiple reviewers showing excellent agreement; however, it is possible that we missed documentation of goals-of-care conversations and ACP and that some conversations may not have been documented. We counted documents completed within 6 months of admission; documents completed after discharge may not be directly related to the SQ response at the time of hospitalization. Both the ED and inpatient admission order sets require an answer to the SQ - however, inpatient providers may opt to use a different order set that does not require an answer to the SQ. Although we found an increased occurrence of ACP in patients who were $\mathrm{SQ}+$, the causal direction of this association cannot be determined given the cross-sectional nature of this study. However, ACP rates may have been augmented by the institutional efforts to increase palliative care consultations in $\mathrm{SQ}+$ patients.

Despite these limitations, our findings suggest that the 30day and 1-year SQ may be useful prognostic tools for patients with COPD and may be associated with increases in ACP. Future studies are needed to understand (1) whether the association we observed is causal, (2) whether the predictive value of the SQ changes depends on the type of provider, (3) whether asking the SQ later during admission or multiple times during hospital stay improves prognostic accuracy, and (4) whether completion of the SQ by multiple providers may improve its prognostic accuracy. Further work is also needed to understand why the SQ is less accurate for COPD than other diseases and whether there are subgroups for whom the SQ would be more predictive, such as older or frail patients with COPD. Finally, further work is needed to develop effective ways of using the SQ response to trigger ACP for patients with COPD. Our findings support the potential value of the SQ in this effort.

Supplementary Information The online version contains supplementary material available at https://doi.org/10.1007/ s11606-020-06512-8.
Acknowledgments: We would like to acknowledge the contributions of Dr. Lisa Almeder who was instrumental in the integration of the surprise question in our electronic medical record system

Corresponding Author: Rebecca N. Hutchinson, MD, MPH; Center for Outcomes Research and Evaluation, Maine Medical Center Research Institute, Portland, ME, USA (e-mail: rhutchinso@mmc.org).

\section{Compliance with Ethical Standards:}

Conflict of Interest: The authors declare that they do not have a conflict of interest.

\section{REFERENCES}

1. Mathers CD, Loncar D. Projections of Global Mortality and Burden of Disease from 2002 to 2030. PLOS Medicine. 2006;3(11):e442.

2. Murray CJL, Lopez AD. Alternative projections of mortality and disability by cause 1990-2020: Global Burden of Disease Study. The Lancet. 1997;349(9064):1498-1504.

3. Elkington $\mathbf{H}$, White $\mathbf{P}$, Addington-Hall J, Higgs R, Pettinari C. The last year of life of COPD: a qualitative study of symptoms and services. Respiratory Medicine. 2004;98(5):439-445.

4. Maurer J, Rebbapragada V, Borson S, et al. Anxiety and Depression in COPD: Current Understanding, Unanswered Questions, and Research Needs. Chest. 2008;134(4, Supplement):43S-56S.

5. Solano JP, Gomes B, Higginson IJ. A Comparison of Symptom Prevalence in Far Advanced Cancer, AIDS, Heart Disease, Chronic Obstructive Pulmonary Disease and Renal Disease. Journal of Pain and Symptom Management. 2006;31(1):58-69.

6. Claessens MT, Lynn J, Zhong Z, et al. Dying with lung cancer or chronic obstructive pulmonary disease: insights from SUPPORT. $J$ Am Geriatr Soc. 2000;48(S1):S146-S153.

7. De Schreye R, Smets T, Deliens L, Annemans L, Gielen B, Cohen J. Appropriateness of End-of-Life Care in People Dying From COPD. Applying Quality Indicators on Linked Administrative Databases. Journal of Pain and Symptom Management. 2018;56(4):541-550.e546.

8. Gore JM, Brophy CJ, Greenstone MA. How well do we care for patients with end stage chronic obstructive pulmonary disease (COPD)? A comparison of palliative care and quality of life in COPD and lung cancer. Thorax. 2000;55(12): 1000-1006.

9. Reinke LF, Slatore CG, Uman J, et al. Patient-clinician communication about end-of-life care topics: Is anyone talking to patients with chronic obstructive pulmonary disease? Journal of palliative medicine. 2011;14(8):923-928.

10. Au DH, Udris EM, Engelberg RA, et al. A randomized trial to improve communication about end-of-life care among patients with COPD. Chest. 2012;141(3):726-735.

11. Janssen DJ, Spruit MA, Schols JM, Wouters EF. A call for high-quality advance care planning in outpatients with severe COPD or chronic heart failure. Chest. 2011;139(5):1081-1088

12. Siouta N, Clement P, Aertgeerts B, Van Beek K, Menten J. Professionals' perceptions and current practices of integrated palliative care in chronic heart failure and chronic obstructive pulmonary disease: a qualitative study in Belgium. BMC Palliat Care. 2018;17(1):103.

13. Bloom CI, Slaich B, Morales DR, Smeeth L, Stone P, Quint JK. Low uptake of palliative care for COPD patients within primary care in the UK. European Respiratory Journal. 2018;51(2):1701879.

14. Murray SA, Kendall M, Boyd K, Sheikh A. Illness trajectories and palliative care. Bmj. 2005;330(7498):1007-1011.

15. Bertens LCM, Reitsma JB, Moons KGM, et al. Development and validation of a model to predict the risk of exacerbations in chronic obstructive pulmonary disease. Int $J$ Chron Obstruct Pulmon Dis. 2013;8:493-499

16. Smith L-JE, Moore E, Ali I, Smeeth L, Stone P, Quint JK. Prognostic variables and scores identifying the end of life in COPD: a systematic review. Int $J$ Chron Obstruct Pulmon Dis. 2017;12:2239-2256.

17. Boeck L, Soriano JB, Brusse-Keizer M, et al. Prognostic assessment in COPD without lung function: the B-AE-D indices. European Respiratory Journal. 2016;47(6):1635-1644.

18. Steer J, Gibson J, Bourke SC. The DECAF Score: predicting hospital mortality in exacerbations of chronic obstructive pulmonary disease. Thorax. 2012;67(11):970. 
19. Fawzy A, Anderson JA, Cowans NJ, et al. Association of platelet count with all-cause mortality and risk of cardiovascular and respiratory morbidity in stable COPD. Respiratory Research. 2019;20(1):86.

20. Aaronson EL, George N, Ouchi K, et al. The Surprise Question Can Be Used to Identify Heart Failure Patients in the Emergency Department Who Would Benefit From Palliative Care. J Pain Symptom Manage. 2019;57(5):944-951

21. Straw S, Byrom R, Gierula J, et al. Predicting one-year mortality in heart failure using the 'Surprise Question': a prospective pilot study. European Journal of Heart Failure. 2019;21(2):227-234.

22. Lakin JR, Robinson MG, Bernacki RE, et al. Estimating 1-Year Mortality for High-Risk Primary Care Patients Using the "Surprise" Question. JAMA Intern Med. 2016;176(12):1863-1865.

23. Ouchi K, Strout T, Haydar S, et al. Association of Emergency Clinicians' Assessment of Mortality Risk With Actual 1-Month Mortality Among Older Adults Admitted to the Hospital. JAMA Network Open. 2019;2(9):e1911139-e1911139.

24. Haydar SA, Strout TD, Bond AG, Han PKJ. Prognostic value of a modified surprise question designed for use in the emergency department setting. Clin Exp Emerg Med. 2019;6(1):70-76.

25. Downar J, Goldman R, Pinto R, Englesakis M, Adhikari NK. The "surprise question" for predicting death in seriously ill patients: a systematic review and meta-analysis. Can Med Assoc J. 2017;189(13):E484-E493.

26. Moss AH, Ganjoo J, Sharma S, et al. Utility of the "surprise" question to identify dialysis patients with high mortality. Clin $J$ Am Soc Nephrol. 2008;3(5):1379-1384.

27. Moss AH, Lunney JR, Culp S, et al. Prognostic significance of the "surprise" question in cancer patients. J Palliat Med. 2010;13(7):837-840.

28. Vick JB, Pertsch N, Hutchings $\mathbf{M}$, et al. The utility of the surprise question in identifying patients most at risk of death. Journal of Clinical Oncology. 2015;33(29_suppl):8-8.

29. Feyi K, Klinger S, Pharro G, et al. Predicting palliative care needs and mortality in end stage renal disease: use of an at-risk register. BMJ Support Palliat Care. 2015;5(1):19-25.

30. Hamano J, Morita $\mathbf{T}$, Inoue $\mathbf{S}$, et al. Surprise questions for survival prediction in patients with advanced cancer: a multicenter prospective cohort study. Oncologist. 2015;20(7):839-844.

31. Pang WF, Kwan BC, Chow KM, Leung CB, Li PK, Szeto CC. Predicting 12-month mortality for peritoneal dialysis patients using the "surprise" question. Perit Dial Int. 2013;33(1):60-66.

32. Rhee J, Clayton JM. The 'surprise' question may improve the accuracy of GPs in identifying death in patients with advanced stage IV solid-cell cancer. Evid Based Med. 2015;20(2):71.
33. Noppe D, Veen Hit, Mooren $\mathbf{K}$. COPD patients in need of palliative care: Identification after hospitalization through the surprise question. Chronic Respiratory Disease. 2019;16:1479972318796219.

34. South G, Reddington O, Hatfield L, Phillips A, Wall H. End of life in COPD: There may be no surprises! European Respiratory Journal. 2011;38(Suppl 55):p1241.

35. https://www.cms.gov/medicare/medicare-fee-for-service-payment/ acuteinpatientpps/readmissions-reduction-program. Accessed 2/20/2020.

36. Harris PA, Taylor R, Thielke R, Payne J, Gonzalez N, Conde JG. Research electronic data capture (REDCap)-a metadata-driven methodology and workflow process for providing translational research informatics support. J Biomed Inform. 2009;42(2):377-381.

37. Keating NL, Huskamp HA, Kouri E, et al. Factors contributing to geographic variation in end-of-life expenditures for cancer patients. Health Aff (Millwood). 2018;37(7):1136-1143.

38. Sudore RL, Lum HD, You JJ, et al. Defining advance care planning for adults: a consensus definition from a multidisciplinary Delphi panel. $J$ Pain Symptom Manage. 2017;53(5):821-832.e821.

39. Edmonds KP, Ajayi TA. Do We Know What We Mean? An Examination of the Use of the Phrase "Goals of Care" in the Literature. Journal of Palliative Medicine. 2019;22(12):1546-1552.

40. Stevenson Mea. epiR: Tools for the Analysis of Epidemiological Data. R package version 1.0-4 . 2019; https://CRAN.R-project.org/package = epiR.

41. Team RC. R: A language and environment for statistical computing. 2019; http://www.R-project.org/, 2020.

42. White N, Kupeli N, Vickerstaff V, Stone P. How accurate is the 'Surprise Question' at identifying patients at the end of life? A systematic review and meta-analysis. BMC Med. 2017;15(1):139.

43. Singh S, Rodriguez A, Lee D, Min S-J, Fischer S. Usefulness of the Surprise Question on an Inpatient Oncology Service. American Journal of Hospice and Palliative Medicine ${ }^{\circledR}$. 2018;35(11):1421-1425.

44. Haydar SA, Almeder L, Michalakes L, Han PK, Strout TD. Using the Surprise Question To Identify Those with Unmet Palliative Care Needs in Emergency and Inpatient Settings: What Do Clinicians Think? J Palliat Med. 2017.

45. Moss AH, Lunney JR, Culp S, et al. Prognostic Significance of the "Surprise" Question in Cancer Patients. Journal of Palliative Medicine. 2010;13(7):837-840.

46. Gerlach C, Goebel S, Weber S, Weber M, Sleeman KE. Space for intuition - the 'Surprise'-Question in haemato-oncology: Qualitative analysis of experiences and perceptions of haemato-oncologists. Palliative Medicine. 2019;33(5):531-540.

Publishers Note Springer Nature remains neutral with regard to jurisdictional claims in published maps and institutional affiliations. 